ESAIM: PROCEEDINGS AND SURVEYS, September 2014, Vol. 45, p. 390-399

J.-S. Dhersin, Editor

\title{
IDENTIFICATION OF MULTIPOLAR SOURCES IN A BIOLUMINESCENT TOMOGRAPHY PROBLEM
}

\author{
Batoul Abdelaziz ${ }^{1}$, Abdellatif El Badia ${ }^{1}$, Ahmad El HajJ ${ }^{1}$
}

\begin{abstract}
Bioluminescence Tomography (BLT) is a recently developed noninvasive imaging tool that allows a direct study of the molecular activity in small animal models. While the forward problem is reduced to a diffusion equation since the scattering phenomena are dominated by the absorption ones in biological tissues, the reconstruction of the distribution of the BLT source is an inverse source problem. In this paper, we concentrate on the reconstruction method where we present an algebraic method allowing to identify the number, the intensities and the location of monopolar sources. Finally, some numerical results are shown proving the robustness of the method.

Résumé. Identification de sources multipolaire dans un problème de tomographie par bioluminescence La Tomographie par Bioluminescence (BLT) est un outil non invasive d'imagerie récemment développé dont l'objectif est d'étudier l'activité moléculaire dans de petits animaux. Étant donnée que dans les tissues biologiques, les phénomenes de dispersion sont dominé par l'absorption, le modèle mathématique sous adjacent est réduit à une équation de diffusion. Le problème inverse en BLT consiste à déterminer de la distribution des sources bioluminescence au moyen de mesure de flux surfacique. Dans le cadre de ce travail, nous considérons des sources multipolaires pour lesquelles nous présentons une méthode d'identification algébrique nous permettant de déterminer leur nombre, leurs intensités et leurs positions. Des résultats numériques sont effectués montrant la robustesse de notre méthode.
\end{abstract}

\section{INTRODUCTION}

Inverse source problems are in the core of many engineering and biomedical applications. In this paper, we concentrate our study over one of the recent developing problems namely the inverse source problem related to bioluminescence tomography (BLT). Let us recall a brief description of BLT. This method is a newly developed technique for molecular imaging allowing in vivo studies on small animals, especially living mice. It is based on the use of luciferase enzyme, responsible for light emission, and consists in detecting the external optical signals measures using a highly sensitive charged-coupled device (CCD) camera. Then, using an optical tomographic study to recover the optical properties of the domain $[4,5]$ and the mouse anatomy, the goal is to reconstruct the 3D distribution of BLT sources representing the reporter cell activity. In fact, BLT is an increasingly important tool for biomedical researchers. It can help diagnose diseases, evaluate and monitor therapies and facilitate drug development with mouse models by allowing real time tomographic localization and monitoring of the disease. Its main application is in the gene therapy where the gene, confined with luciferase, is injected in the mouse. The BLT technique is employed to reconstruct the reporter gene activity distribution.

\footnotetext{
${ }^{1}$ Laboratory of Applied Mathematics, LMAC, University of Technology of Compiègne, UTC, France, e-mail: batoul.abdelaziz@utc.fr, abdellatif.elbadia@utc.fr, ahmad.el-hajj@utc.fr
}

(C) EDP Sciences, SMAI 2014 
In a mathematical point of view, the inverse BLT source problem aims to reconstruct, localize and quantify the $3 D$ bioluminescent source distribution inside a domain based on external bioluminescent optical signals measures. The forward problem is characterized, in a specific framework, by a stationary diffusion equation with a pointwise source term which may represent the early stage of a tumor development and thus helps in the early diagnosis of a cancer. The inverse problem, we are interested in, is to recover the source term from the Cauchy data pair obtained over the boundary of the domain. The statement and the modeling issues are presented in Section 1. Then, in Section 2, we propose a direct and non-iterative algebraic method used for the identification of the number, the intensity and the location of these sources. Finally, we present in Section 3, the numerical results that show the stability of the identification method with respect to the different parameters interfering in the reconstruction process.

\section{Statement AND MOdeling of THE PROBlem}

The basic equation governing the light migration in a random medium is the Radiative Transfer Equation (RTE), also known as Boltzmann equation. However, since in biological tissues [16,17], the mean-free path of the particle is between 0.005 and $0.01 \mathrm{~mm}$, the scattering phenomenon dominates on the absorption phenomenon and thus the transport equation is approximated by a diffusion equation [4]. Moreover, since the internal bioluminescent distribution induced by the reporter genes is relatively stable, we consider the stationary diffusion equation where the photon fluence rate $u=u(x)$ satisfies the system:

$$
\begin{cases}\nabla \cdot(D(x) \nabla u)(x)+\mu_{a}(x) u(x)=F(x) & \text { for } \quad x \in \Omega \subset \mathbb{R}^{3} \\ u(x)+2 D(x) \frac{\partial u}{\partial \nu}(x)=g^{-}(x) & \text { for } \quad x \in \Gamma\end{cases}
$$

The source term $F$ represents the bioluminescent source distribution and $D(x)=\frac{1}{3\left(\mu_{a}(x)+\mu_{s}^{\prime}(x)\right)}$ where $\mu_{a}(\cdot)$ and $\mu_{s}^{\prime}(\cdot)$ are consecutively the absorption and the reduced scattering coefficients. The function $g^{-}$represents the incoming flux which is, in a typical BLT, identically null since the experiment is performed in a totally dark environment and no external photon travels into $\Omega$ through its boundary $\Gamma$ and $\nu$ denotes the outward normal vector to $\Omega$.

The aim of inverse BLT problem is to reconstruct the internal bioluminescent source $F$ using the external optical measures that is the flux on the boundary $g$, given by

$$
g(x)=-D(x) \frac{\partial u}{\partial \nu}(x) \quad x \in \Gamma .
$$

The boundary condition and the measurement can be added to get the Cauchy data pair $\left(u_{\left.\right|_{\Gamma}},\left.\frac{\partial u}{\partial \nu}\right|_{\Gamma}\right)$ where

$$
u(x)=g^{-}(x)+2 g(x) \quad:=f \quad x \in \Gamma
$$

In the following framework, we consider the case of a body consisting of several interior organs (heart, lungs, ...) where each organ is assumed to be homogenous (in an optical point of view). This leads to consider $\Omega$ as a bounded, simply connected domain in $\mathbb{R}^{3}$ with sufficiently regular boundary $\Gamma$ consisting of doubly connected disjoint subdomains $\Omega_{i}, i=1, . ., m$ and their respective boundaries $\Gamma_{i}$ (see Figure 1). The homogeneity in each $\Omega_{i}$ amounts to assuming that $D$ and $\mu_{a}$ are constant in each subdomain and accordingly are of the form

$$
D=\sum_{i=1}^{m} D^{i} \chi_{\Omega_{i}}, \quad \mu_{a}=\sum_{i=1}^{m} \mu_{a}^{i} \chi_{\Omega_{i}}
$$

where $\chi_{\Omega_{i}}$ denotes the characteristic function of the domain $\Omega_{i}$ and $D^{i}$ and $\mu_{a}^{i}$ are respectively positive and negative constants. 


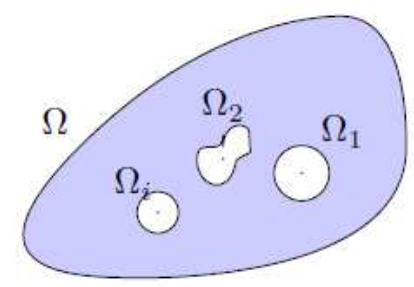

Figure 1. Domain shape.

Supposing that the source $F$ is localized in one of the interior subdomains, for example $\Omega_{1}$, our aim is to reconstruct it using the boundary measurements. Note that in order to recover the Cauchy data pair $\left(u_{\left.\right|_{\Gamma_{1}}},\left.\frac{\partial u}{\partial \nu}\right|_{\Gamma_{1}}\right)$ over the desired subdomain, it is sufficient to solve a Cauchy problem which numerically stays reasonable especially that the considered domain is relatively small.

To simplify the presentation in the rest of the paper, we denote $\Omega_{1}, \Gamma_{1},\left(u_{\left.\right|_{\Gamma_{1}}},\left.\frac{\partial u}{\partial \nu}\right|_{\Gamma_{1}}\right), \mu_{a}^{1}$ respectively by $\Omega, \Gamma$, $(f, g), \mu$ and we take $D^{1}=1$, which brings us back to the study of the following problem

$$
\Delta u+\mu u=F \quad \text { in } \quad \Omega, \quad \text { with } \quad \mu \leq 0 .
$$

One of the major difficulties of the inverse source problem from boundary measurements is the problem of uniqueness. As proven in [17], in the general case where the source $F \in L^{2}(\Omega)$, the source reconstruction is not unique. To overcome this difficulty, we assume that some a priori information on the source are available, depending on the underlying physical problem. In [12], the sources, in a Helmoltz equation were of either the form $F=\rho(x) \chi_{B}(x)$ where $B$ is an open subset of $\Omega, \quad \chi_{B}$ is the characteristic function of $B$, or the form $F=\operatorname{div}\left[\rho(x) \chi_{B}(x) a\right]$, where $a$ is a nonzero constant vector. Under additional conditions the convex hull of $B$ was reconstructed using the cauchy data. The reconstruction of extended sources for the 2D Helmholtz equation is studied in [13]. One can also mention, in the case of the exterior Helmholtz problem, the paper [3] concerning monopolar sources having a known number where an iterative scheme was proposed. The case of dipolar sources have been considered in [10] in the case of Poisson equation $(\mu=0)$ because of their interest in the inverse EEG/MEG problem. This work has been revisited in $[6,14]$ considering combination of monopoles and dipoles and recently in $[7,15]$ considering sources of general order poles. The proposed algorithms in the previous papers are based on the invertibility of a Hankel-type matrix $H$, using the calculation of its determinant. This is very long and tedious. In contrast, our method presented in [1] is more general and simpler, where we have considered the case of a source of multipolar pointwise form

$$
F=\sum_{\ell=1}^{L} \sum_{j=1}^{N^{\ell}} \sum_{\alpha=0}^{K^{\ell}} \lambda_{j, \ell}^{\left\{\alpha_{1}, \alpha_{2}, \alpha_{3}\right\}} \frac{\partial^{\alpha}}{\partial_{x}^{\alpha_{1}} \partial_{y}^{\alpha_{2}} \partial_{z}^{\alpha_{3}}} \delta_{\mathbf{S}_{j}^{\ell}}
$$

where $\delta_{\mathbf{S}}$ stands for the Dirac distribution at point $\mathbf{S}$, the quantities $L, N^{\ell}, K^{\ell}$ are integers and $\alpha=\alpha_{1}+\alpha_{2}+\alpha_{3}$ with $\left(\alpha_{1}, \alpha_{2}, \alpha_{3}\right) \in \mathbb{N}^{3}$. The points $\mathbf{S}_{j}^{\ell}=\left(x_{j}^{\ell}, y_{j}^{\ell}, z_{j}^{\ell}\right)$ belong to $\Omega$ and are assumed to be mutually distinct. The orders of derivation $K^{\ell}$ are also assumed to be mutually distinct and the coefficients $\lambda_{j, \ell}^{\left\{\alpha_{1}, \alpha_{2}, \alpha_{3}\right\}}$ are scalar quantities. It is rather interesting to point out, that by the means of Taylor expansion, the case with small inclusion sources follows that with sources of form (3) as seen in [2]. Then, the inverse BLT problem is brought back to the problem of determining the number of sources $N^{\ell}$, their locations $\mathbf{S}_{j}^{\ell}$ and the coefficients $\lambda_{j, \ell}^{\left\{\alpha_{1}, \alpha_{2}, \alpha_{3}\right\}}$. To be more precise, we begin by defining the application

$$
\Lambda: F \rightarrow\left(u_{\left.\right|_{\Gamma}},\left.\frac{\partial u}{\partial \nu}\right|_{\Gamma}\right) .
$$


Then, given the source $F$ of the form (3), the direct problem is well-posed and thus the Cauchy pair $\left(u_{\left.\right|_{\Gamma}},\left.\frac{\partial u}{\partial \nu}\right|_{\Gamma}\right)$ are well-defined in the $H^{\frac{1}{2}}(\Gamma) \times H^{-\frac{1}{2}}(\Gamma)$ space. Hence, the inverse problem, we are concerned with, is formulated as follows:

$$
\text { Given }(f, g) \in H^{\frac{1}{2}}(\Gamma) \times H^{-\frac{1}{2}}(\Gamma), \quad \text { determine } F \text { such that } \quad \Lambda(F)=(f, g) .
$$

In order to present the idea behind the algebraic method that serves as an identification process, we chose to treat, in the following section, the particular case of monopolar sources. The general case considering multiple sources is discussed thoroughly in [1].

\section{Algebraic identification method}

The case of monopoles represents the case of sources $F$ satisfying (3) with $L=1$ and $K^{1}=0$, namely,

$$
F=\sum_{j=1}^{N} \lambda_{j} \delta_{\mathbf{S}_{j}}
$$

Thus, the goal is to identify the number of point sources $N=N^{1}$, their intensities $\lambda_{j}$, and their positions $\mathbf{S}_{j}$ given the Cauchy data pair $(f, g)$. Compared to optimization-based iterative algorithms for inverse problem, the algebraic method has an advantage that it doesn't require the initial solution and iterative computation of the forward problem. From the practical viewpoint, the solution obtained by our method can be used as an initial solution to the iterative algorithm, which is quite important to prevent it from converging to the local minimum. Uniqueness issue is trivial using Holmgrem's theorem and the regularity of the direct problem. The stability in given in $[8,9]$ where it is proven that the error in localization reconstruction depends not only on the number of monopoles but also many other effects as the separability between the sources, the distance from the boundary and also the coefficient $\mu$. These effects are studied and verified numerically in Section 3 . Before formulating the algorithm, we introduce some notations and specify additional information.

First, we introduce the space of the homogenous equation in $\Omega$ :

$$
\mathcal{H}_{\mu}=\left\{v \in \mathrm{H}^{1}(\Omega): \Delta v+\mu v=0\right\}
$$

and define the operator $\mathcal{R}$ as follows

$$
\mathcal{R}(v, f, g)=\int_{\Gamma}\left(g v-f \frac{\partial v}{\partial \nu}\right) d s \quad \text { for all } \quad v \in \mathcal{H}_{\mu}
$$

Multiplying equation (2) by $v$ element of $\mathcal{H}_{\mu}$, integrating by parts and then using Green formula gives

$$
\mathcal{R}(v, f, g)=\sum_{j=1}^{N} \lambda_{j} v\left(\mathbf{S}_{j}\right) \quad \text { for all } \quad v \in \mathcal{H}_{\mu} .
$$

Choosing, for $n \in \mathbb{N}$, the test functions

$$
v_{n}(x, y, z)=(x+i y)^{n} e^{k z} \quad \text { with } \quad k^{2}=-\mu,
$$

one can observe that $v_{n} \in \mathcal{H}_{\mu}$. Then, we set

$$
c_{n}=\mathcal{R}\left(v_{n}, f, g\right),
$$


define, for $\bar{N} \in \mathbb{N}^{*}$, the complex Hankel matrix

$$
H_{\bar{N}}=\left(\begin{array}{llll}
c_{0} & c_{1} & \cdots & c_{\bar{N}-1} \\
c_{1} & c_{2} & \cdots & c_{\bar{N}} \\
\vdots & \vdots & \vdots & \vdots \\
c_{\bar{N}-1} & c_{\bar{N}} & \cdots & c_{2 \bar{N}-2}
\end{array}\right)
$$

and introduce $T$ the Companion matrix:

$$
T=\left(\begin{array}{lllll}
0 & 1 & \cdots & 0 & 0 \\
0 & 0 & 1 & \cdots & 0 \\
\vdots & \vdots & \ddots & \ddots & 0 \\
\vdots & \vdots & \ddots & \ddots & 1 \\
b_{0} & b_{1} & \cdots & \cdots & b_{N-1}
\end{array}\right)
$$

where the vector $b=\left(b_{0}, \ldots, b_{N-1}\right)^{t}$ is obtained by solving the linear system $H_{N} b=\xi_{N}$ with $\xi_{N}=\left(c_{N}, \ldots, c_{2 N-1}\right)^{t}$.

Consider the mentioned assumptions on the sources and suppose that the number of monopoles $N$ is upper bounded by a non-negative integer $\bar{N}$, then our identification algorithm relies on the following result proved in [10] and also in [1] considering the general case where $F$ is given by (3).

(1) Let $H_{\bar{N}}$ be the Hankel Matrix defined in (5), then

$$
\operatorname{rank}\left(H_{\bar{N}}\right)=N
$$

(2) The eigenvalues of the Companion matrix $T$, defined in (6), are the $2 D$ projections on the $x y$-complex plane of the sources $\mathbf{S}_{j}$, for $j=1, \cdots, N$.

The previous result allows us to determine the $2 D$ projection on the $x y$ plane of the point sources. In order to determine their projected points on $x z$ and $y z$ planes, we do the same thing using the following test functions

$$
\bar{v}_{n}(x, y, z)=(x+i z)^{n} e^{k y}, \quad \tilde{v}_{n}(x, y, z)=(y+i z)^{n} e^{k x} .
$$

In the following section, we will test this method numerically in order to show the effect of the several parameters (number, absorption coefficient, noise,...) on the reconstruction.

\section{Numerical Results}

In this section, we study numerically the robustness of the algebraic algorithm mentioned above with respect to the different parameters interfering in the reconstruction process, where we show their coherence with the theoretical stability estimate obtained in [9] and recalled in (7). The main theorem related to the source position reconstruction obtained in [9] states that, if $u^{\ell}$ for $\ell=1,2$ are the solutions of (2) corresponding to two sources characterized by the configuration $\mathbf{S}_{j}^{\ell}$, then there exists three constants $c, c_{1}, c_{2}$ and a permutation $\pi$ of the integers $1, \ldots, m$ such that

$$
\max _{1 \leq j \leq N}\left\|\mathbf{S}_{j}^{2}-\mathbf{S}_{\pi(j)}^{1}\right\| \leq 2 c \frac{\beta^{2}}{\varrho}\left[\frac{\sqrt{|\Gamma|}}{c_{1}} \frac{\varrho}{\beta}\left[\left\|g^{2}-g^{1}\right\|_{L^{2}(\Gamma)}+c_{2}\left\|f^{2}-f^{1}\right\|_{L^{2}(\Gamma)}\right]\right]^{\frac{1}{N}}
$$


where $\left(f^{\ell}, g^{\ell}\right)=\left(u_{\left.\right|_{\Gamma}}^{\ell},\left.\frac{\partial u^{\ell}}{\partial \nu}\right|_{\Gamma}\right), \sqrt{|\Gamma|}=\int_{\Gamma} d s, \beta$ is the distance of the sources from the boundary and $\varrho$ is the separability coefficient between the sources defined consecutively by:

$$
\beta=\operatorname{diam}(\Omega)-\alpha, \quad \text { where } \quad \alpha=\min _{1 \leq j \leq N} d\left(\Gamma, \mathbf{S}_{j}\right)
$$

and

$$
\varrho=\min \left(\varrho_{1}, \varrho_{2}\right), \quad \text { where } \varrho_{1}=\min _{1 \leq j, n \leq N, j \neq n}\left\|P_{j}-P_{n}\right\| \quad \text { and } \varrho_{2}=\min _{1 \leq j, n \leq N, j \neq n}\left\|Q_{j}-Q_{n}\right\|
$$

with $P_{j}$ and $Q_{j}$ are respectively the $x y$ and $y z$ projections of $\mathbf{S}_{j}$.

Remark 3.1. We note that in [9], the authors considered only the case with $\mu \geq 0$, where (7) was obtained with $c=1$. However, in the case where $\mu<0$, one can obtain in the same manner the theoretical stability estimate mentioned above but with a positive constant $c>1$.

From (7), one can see that several factors such as the number of sensors, the separability coefficient, the noise and the absorption coefficient $\mu$ have an important effect on the stability of the identification process. In the following subsections, the effect of these parameters will be studied numerically.

\subsection{Determining number and position of sources}

In this subsection, we aim to reconstruct monopoles whose intensities are supposed to be the same $\lambda_{j}=1$, the absorption coefficient is fixed at $\mu=-3.42$ and $\Gamma$ is assumed to be a unit sphere whose center is the origin $O$. The Cauchy data $(f, g)$ on the boundary $\Gamma$ are computed using the fundamental solution in $\mathbb{R}^{3}$ over a uniforming meshing of distributed points on the unit sphere using spherical coordinates. Starting with $25^{2}$ sensors over the domain's boundary, we were able to identify the number and $2 D$ location of up to 4 monopoles. Indeed, this is shown in Figure 2 and Figure 3 that presents the singular values for the Hankel matrices $H_{\bar{N}}$ for the 2D $x y$ projection for 4 and 5 monopoles and their corresponding projected positions respectively. We can notice that using the convenient threshold and noting the occurrence of the large gap in the singular value distribution of $H_{\bar{N}}$, the number and the precise localization of 4 monopoles can be well-approximated for $25^{2}$ mesh points while this isn't the case for more than 5 monopoles. This validates that the accuracy in the monopoles localization and their number identification decrease as the number of monopoles increases which is consistent with the estimate (7).

On the other hand, if one possesses more sensors, for instance $50^{2}$ sensors, the 5 monopoles can be well approximated as shown in Figure 4. By increasing gradually the number of sensors, we can reconstruct precisely the number and the position up to 10 monopoles. The latter is obtained when using $100^{2}$ sensors (see Figure 5). Although, as we have tested, meshing more finely leads also to identify much more than 10 monopoles, a higher number of sensors becomes "unrealistic".
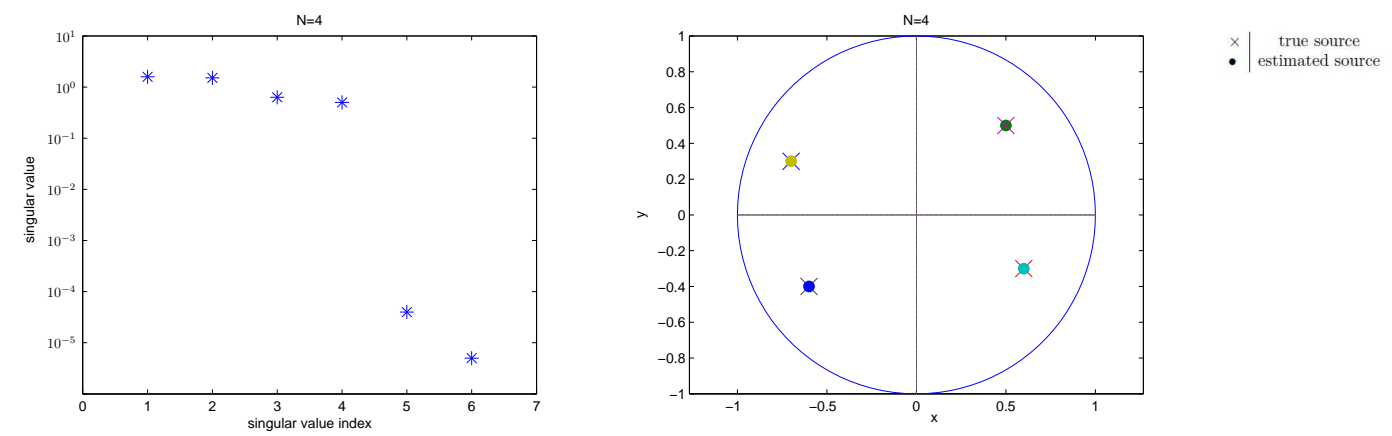

FiguRE 2. Singular values of $H_{\bar{N}}, \bar{N}=6$ and the localization results projected on the $x y$ plane for $N=4$ with $25^{2}$ sensors. 

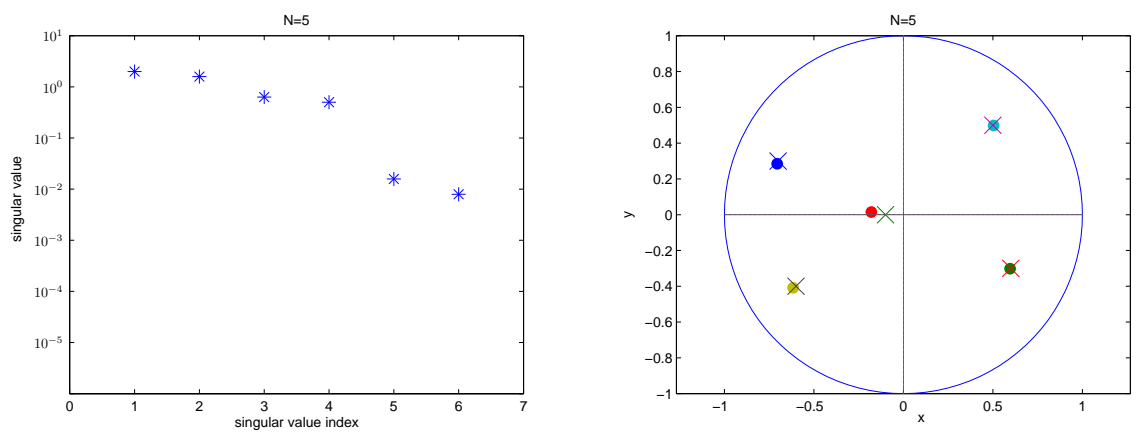

\begin{tabular}{l|c} 
- & true source \\
estimated source
\end{tabular}

FIGURE 3. Singular values of $H_{\bar{N}}, \bar{N}=6$ and the localization results projected on the $x y$ plane for $N=5$ with $25^{2}$ sensors.
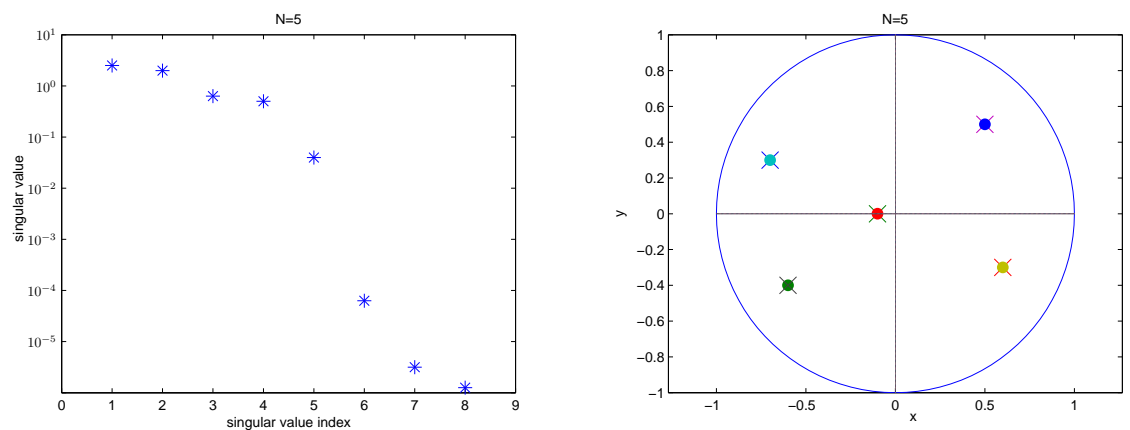

\begin{tabular}{l|c}
$\times$ & true source \\
. & estimated source
\end{tabular}

FIGURE 4. Singular values of $H_{\bar{N}}, \bar{N}=8$ and the localization results projected on the $x y$ plane for $N=5$ with $50^{2}$ sensors.
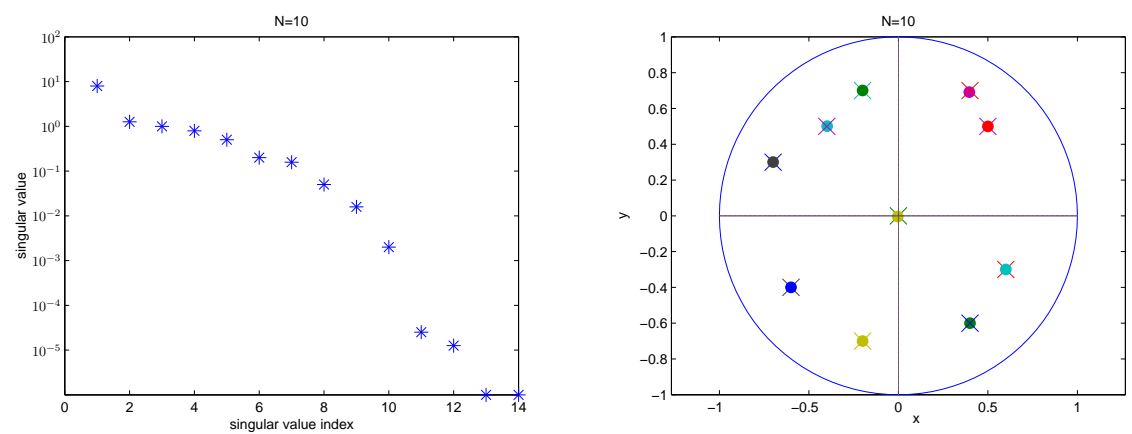

\begin{tabular}{c|c}
$\times$ & true source \\
- & estimated source
\end{tabular}

FIGURE 5. Singular values of $H_{\bar{N}}, \bar{N}=14$ and the localization results projected on the $x y$ plane for $N=10$ with $100^{2}$ sensors.

As mentioned before, the numerical results can be enhanced using an important number of sensors which isn't always trivial since the number of sensors is limited. Hence, noting that the projected separability coefficient plays a role in the estimation stability as seen in (7), a good technique to obtain a better estimation is to choose the best projection plane that leads to the highest separability coefficient. Therefore, fixing the number of sensors to $35^{2}$ sensors, the following subsection consists to find the best projection that enables us to recuperate the $3 D$ exact reconstruction of the sources taking into consideration the separability coefficient between them. 
Remark 3.2. We draw the attention of the reader to the fact that in the case of $35^{2}$ sensors, the numerical error can be seen as noise equivalent to $6 \%$ perturbation. Thus, according to (7), the separability coefficient still plays a role in the identification of the sources. However, when increasing the number of sensors to $100^{2}$ sensors, equivalent to $2 \%$ noise, this error is negligible with respect to the separability coefficient. Therefore, the latter wouldn't have a great influence on the source reconstruction, which justifies the choice of just $35^{2}$ sensors.

\subsection{Obtaining the $3 \mathrm{D}$ coordinates and the effect of the separability coefficient}

To obtain the $3 D$ coordinates of the sources, we use consequently the test functions projected on the $2 D$ planes as shown in Figure 6 and Figure 7 in the case of 5 monopoles. Note that, theoretically the number of sources must be the same whatever the complex plane onto which the projections are performed (distinct projections). However, numerically the situation may be different since the number depends also on the separability of these projections. This is, also, observed in the stability estimate (7). Therefore, it is preferable to calculate the rank of the three consecutive Hankel matrices corresponding to the test functions $v, \bar{v}, \tilde{v}$ and then we consider the number of sources as their maximum.
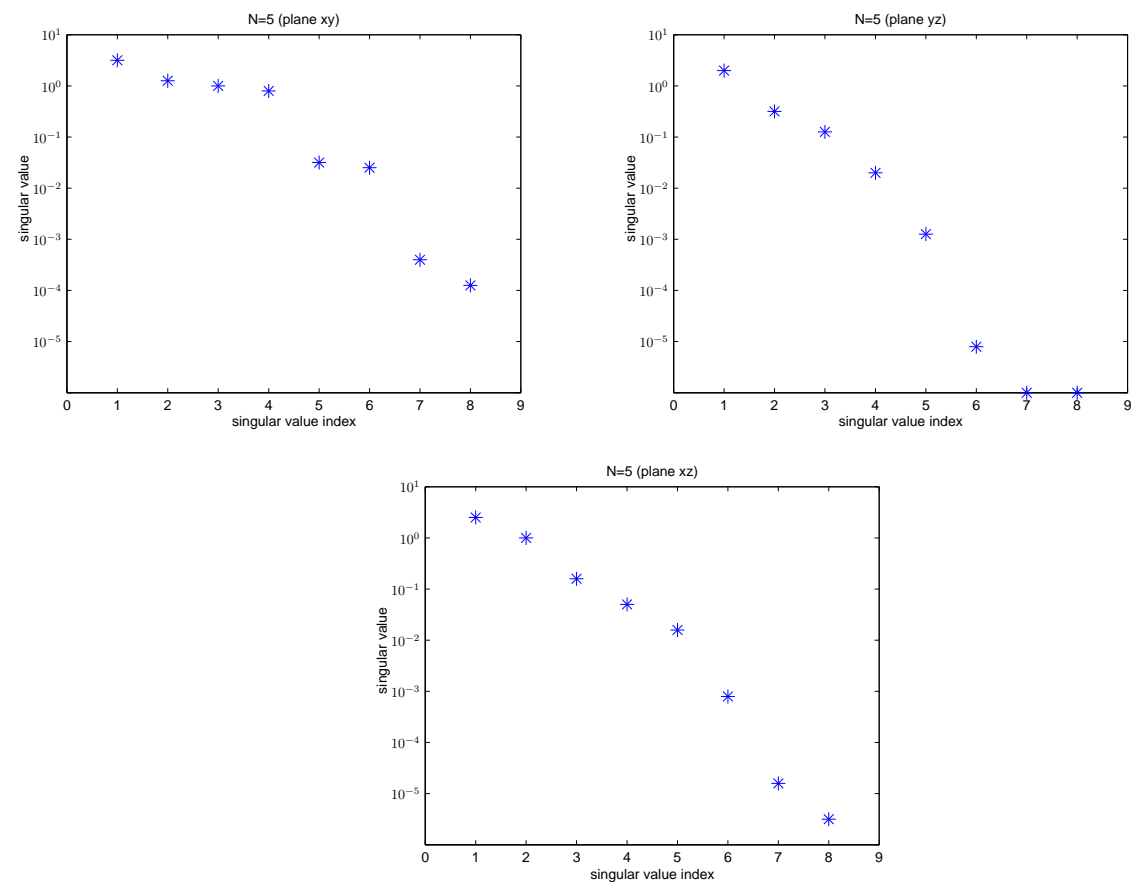

FiguRE 6. Singular values of $H_{\bar{N}}, \bar{N}=8$ for $N=5$ in the $x y, y z$ and $x z$ planes. 

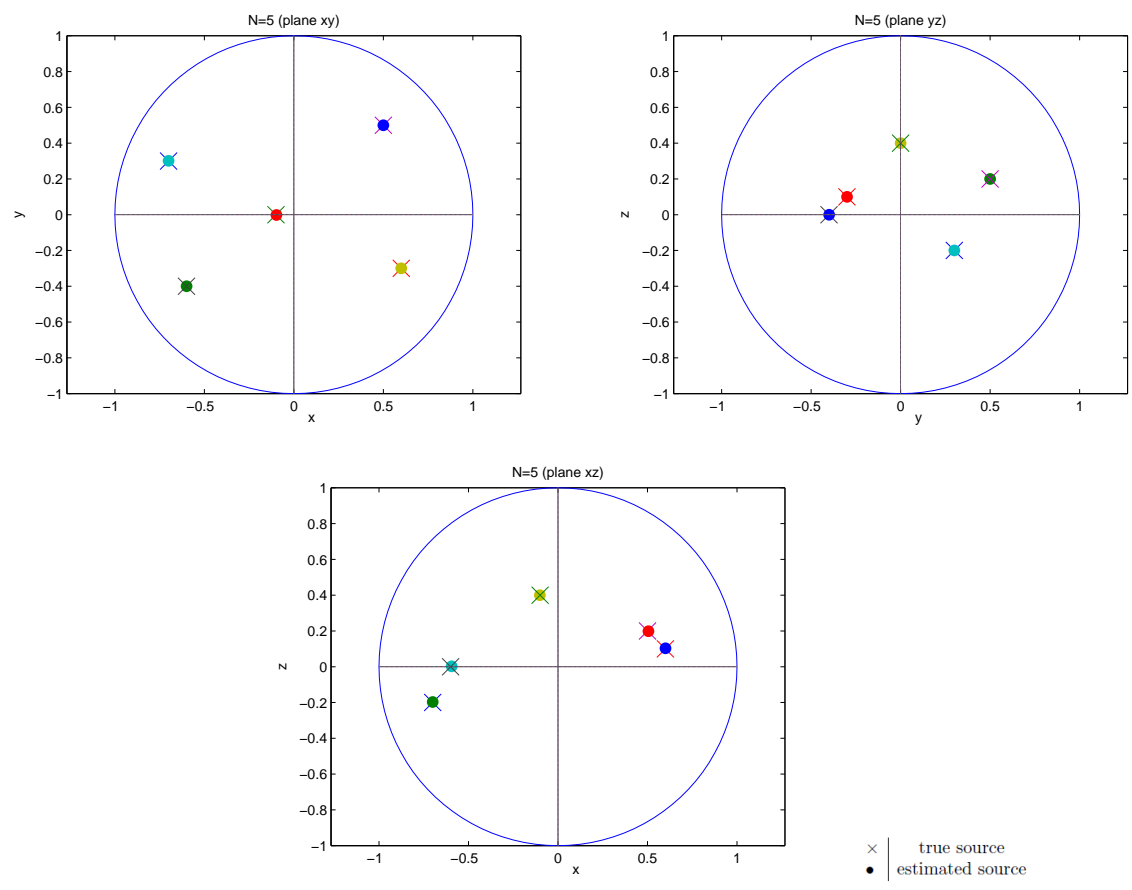

FiguRE 7. Localization results for $N=5$ in the $x y, y z$ and $x z$ planes.

\subsection{Error using Gaussian noise}

Reconstruction stability on the $x y$ projections for 3 monopoles with respect to the noise level is examined in Figure 8. In fact, Gaussian noise is added to $f$ (and $g$ ) where the noise standard deviation added varies from $10^{-4}$ to $10^{0} \%$. We note that the localization error increases as the percentage of the noise added increases and the curves show a Hölder form, consistent with the stability estimates (7).

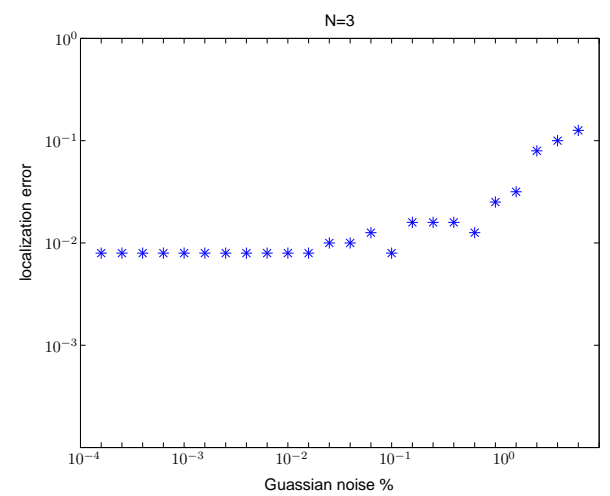

FiguRE 8. The maximum localization error with respect to the noise level for $N=3$.

\subsection{Effect of changing $\mu$}

Finally, we study the effect of changing $\mu$ on the localization accuracy. As shown in Figure 9, where we change $\mu$ in the study of 3 monopoles, the localization error increases with respect to $\mu$ and is almost linear. 
This is consistent with the stability result (7) and this shows that in practice, taking a small enough absorption coefficient leads to a better estimation. Moreover, this phenomenon with negative $\mu$ stands for diffusion rather than propagation that uses positive coefficient $\mu$ (corresponding to the wave number). In comparison with the results obtained in $[1,11]$, we notice that the increase in the negative $\mu$ increases the localization error more dramatically and faster. Note that, in Figure 9, we have also presented the effect of the number of sensors on the localization estimation which proves that the finer mesh improves the reconstruction process.

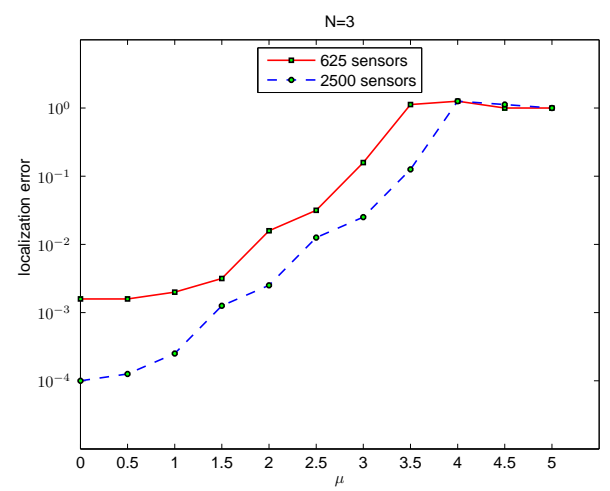

FIGURE 9. The maximum localization error when changing $\mu$ for $N=3$.

\section{REFERENCES}

[1] B. Abdelaziz, A. EL Badia, And A. El HajJ, Reconstruction method for solving some inverse source problems in an elliptic equation from a single Cauchy data, submitted, (2013).

[2] B. Abdelaziz, A. El Badia, ANd A. El HajJ, Reconstruction of extended sources with small supports in the elliptic equation $\Delta u+\mu u=F$ from a single Cauchy data, Comptes Rendus Mathematique, 351 (2013), pp. 797-801.

[3] C. Alves, R. Kress, and P. Serranho, Iterative and range test methods for an inverse source problem for acoustic waves, Inverse Problems, 25 (2009), pp. 055005, 17.

[4] S. R. Arridge, Optical tomography in medical imaging, Inverse Problems, 15 (1999), pp. R41-R93.

[5] S. R. Arridge, O. Dorn, V. Kolehmainen, M. Schweiger, And A. Zacharopoulos, Parameter and structure reconstruction in optical tomography, in Journal of Physics: Conference Series, vol. 135, IOP Publishing, 2008, p. 012001.

[6] Y.-S. Chung And S.-Y. Chung, Identification of the combination of monopolar and dipolar sources for elliptic equations, Inverse Problems, 25 (2009), pp. 085006, 16.

[7] Y.-S. Chung, J. E. Kim, And S.-Y. Chung, Identification of multipoles via boundary measurements, European J. Appl. Math., 23 (2012), pp. 289-313.

[8] A. El Badia And A. El HajJ, Hölder stability estimates for some inverse pointwise source problems, Comptes Rendus Mathematique, 350 (2012), pp. 1031-1035.

[9] - Stability estimates for an inverse source problem of Helmholtzs equation from single Cauchy data at a fixed frequency, Inverse Problems, 29 (2013), p. 125008.

[10] A. El Badia and T. Ha-Duong, An inverse source problem in potential analysis, Inverse Problems, 16 (2000), pp. $651-663$.

[11] A. El Badia And T. NARA, An inverse source problem for Helmholtz's equation from the Cauchy data with a single wave number, Inverse Problems, 27 (2011), pp. 105001, 15.

[12] M. Ikehata, Reconstruction of a source domain from the Cauchy data, Inverse Problems, 15 (1999), pp. $637-645$.

[13] R. KRess and W. Rundell, Reconstruction of extended sources for the Helmholtz equation, Inverse Problems, 29 (2013), p. 035005.

[14] T. NARA, An algebraic method for identification of dipoles and quadrupoles, Inverse Problems, 24 (2008), pp. $025010,19$.

[15] - Algebraic reconstruction of the general-order poles of a meromorphic function, Inverse Problems, 28 (2012), pp. 025008, 19.

[16] F. Natterer and F. Wubbeling, Mathematical methods in image reconstruction, Society for Industrial and Applied Mathematics, Philadelphia, PA, USA, 2001.

[17] G. Wang, Y. Li, AND M. JiAng, Uniqueness theorems in bioluminescence tomography, Medical Physics, 31 (2004), pp. 22892299. 